\title{
Breastfeeding in the transgender; recent advances
}

\begin{abstract}
The studies highlighted in this article, though would be welcome news for the transgender and their campaigners, however, it would further polarize or even alarm some religious, traditional and cultural societies. Some of the latter groups would argue that the time and money put in research for the use of feminising hormones and preparing mammary glands for the transgender could be used to support research to prevent and cure debilitating genetic diseases and other serious medical conditions that are currently blighting humanity. In this article the author reviews recent evidence on breastfeeding in this group of people and outlines ethical and other hurdles before a wider application of the emerging evidence would become a reality.
\end{abstract}

Volume 9 Issue 5 - 2018

\author{
Anthony Emeka Madu \\ Obstetrics and Gynecology, RCOG/FSRH/ILM, UK
}

Correspondence: Anthony Emeka Madu, Specialist Registrar, Obstetrics and Gynecology, RCOG/FSRH/ILM, UK, Tel +44-793162-63I5, Email emymodu@yahoo.co.uk

Received: July 12, 2018 | Published: October 12, 2018

\section{The Case}

Reproductive science has again gone a step further, and in this paper the author analysis the case of a transgender woman in the USA, who after receiving feminising hormone treatment for years, was reported to have later received lactational treatment for three and half years. This treatment enabled her to breastfeed the baby of her female partner. The feminizing treatment she received involved the following regime:

a. Spironolactone; a potassium-sparing diuretic which blocks the effect of testosterone

b. Progesterone

c. Oestrogen (a type of oestrogen)

This regime enabled significant breast development for lactation to take place following further treatment. She did not carry the pregnancy but her partner did and she decided to breastfeed as per sharing the role of raising their baby. It is established in physiology that prolactin stimulates the production of milk in females after they had given birth. Prolactin is however, not available as a laboratorymade drug, thus prompting researchers to use domperidone, an anti-nausea medication, as an unlikely substitute. This lactational treatment comprised of the following regime: A structured regime of domperidone with increasing dose of oestrogen, progesterone, spironolactone, and breast pump stimulation which resulted in the expression of milk droplets in about a month.

After three months, there was increase in expression of breast milk to a peak daily output of $227 \mathrm{~g}$ of milk per day for the first six week, and she breastfed exclusively for 6 weeks. The baby was reported as normal and health (Reisman and Goldstein, 2018).

This output was much lower than the average of $500 \mathrm{~g}$ of milk per day a baby consumes in its first 5 days of life but has been reported as significant. The woman was reported to have supplemented breastfeeding with formula feeds after 6 weeks of breastfeeding. This report appears to be encouraging for the following reasons:

i. If the treatment is proven to be safe and effective, it could benefit this group of parents.

ii. There is therefore potential for breastfeeding for these parents as breast stimulation was reported to have been sufficient for breastfeeding for the infant for the first 6 weeks of its life.

iii. It would also benefit women who chose to breastfeed.

iv. It would also benefit women who have difficulties with initiating and sustaining breast feeding but want to breast feed.

There are valid criticisms of this development and this includes the following:

A. The study involved only one transgender woman and thus does not have sufficient strength to lead to strong conclusions that can lead to wider change of practice based on the study.

B. It is unclear the extent of drugs, medications and hormonal treatments had helped in breast development, breast milk initiation and breast milk sustenance in this research compared to other factors that may have been at play, such as psychological state of the woman subject.

C. The breast milk expressed has not been well assessed so that its contents and proportion of its contents can be compared with breast milk of new non-transgender gestational mothers. Thus, the practice cannot be recommended on this ground alone.

D. The long-term impact on the baby, its immunological and neurological development, including intelligence quotient are still not yet fully evaluated.

It is well established that normal breast milk contains substances necessary for proper immunological and neurological development of the baby, among other aspects of child's development (Hamzelou, J, 2018).

In 2013, in a related and relevant development the UK's National Health Service ruled it would offer eligible same sex female couple's intrauterine insemination (IUI) with sperm donor and if this fails, in vitro fertilization (IVF) would be offered.

\section{Conclusion}

These developments in this area of research are intriguing and encouraging. However, there is still a long way to go before the outcome of this research is put into wider use. There are also ethical hurdles and wider acceptability to overcome. 


\section{Acknowledgements}

None.

\section{Conflict of interest}

The author declares that they have no conflict of interest.

\section{References}

1. Reisman T, Goldstein Z. Case Report: Induced Lactation in a Transgender Woman. Transgend Health. 2018;3(1):24-26.

2. Hamzelou Jessica. Transgender woman is first to be able to breastfeed her baby. NewScientist; 2018. 